\title{
Shifts in the balance of brain tryptophan metabolism due to age and systemic administration of lipopolysaccharide
}

\author{
Hideki Miura', Tetsuya Shirokawa ${ }^{2}$, Norio Ozaki ${ }^{1}$, Kenichi Isobe $^{3}$ \\ ${ }^{1}$ Department of Psychiatry, Nagoya University Graduate School of Medicine, Nagoya, Japan; hmiura@med.nagoya-u.ac.jp \\ ${ }^{2}$ Department of Information Technology for Human Welfare, Nihon Fukushi University, Mihama, Japan \\ ${ }^{3}$ Department of Immunology, Nagoya University Graduate School of Medicine, Nagoya, Japan
}

Received 9 November 2009; revised 22 December 2009; accepted 24 December 2009.

\begin{abstract}
The kynurenine (KYN) pathway, which is initiated by indoleamine 2, 3-dioxygenase (IDO), is a key tryptophan (TRP) metabolic pathway. It shares TRP with the serotonin (5-HT) pathway. Because activation of the KYN pathway by proinflammatory cytokines induces depressive symptoms, shifts in the balance of TRP metabolism to the KYN pathway are closely related to the etiology of depression. In the present study, the influence of age on the effect of the inflammation response system (IRS) on brain TRP metabolism was investigated. Male ICR mice (PND21) were reared for 4 weeks (younger group) or until they reached 1 year of age (older group), and given an intraperitoneal (i.p.) injection of lipopolysaccharide (LPS). The TRP, KYN, and 5-HT levels were measured in the prefrontal cortex, hippocampus, amygdala, and dorsal raphe nuclei. An increase in TRP and 5-HT levels was observed with age in all brain regions, whereas age was associated with decreases in KYN levels in the dorsal raphe nuclei. In all brain regions, LPS increased TRP levels, while it increased KYN levels in the prefrontal cortex and amygdala. Reduced KYN/5-HT ratios in all regions were observed with age, whereas increased KYN/5-HT ratios were observed with LPS in all regions except the dorsal raphe nuclei. Thus, age shifted the balance between the KYN and 5-HT pathways toward the 5-HT pathway, and countered the effects of LPS, which shifted the balance to the KYN pathway. These effects are relevant to the etiology of psychiatric disorders in elderly people.
\end{abstract}

Keywords: Tryptophan; Kynurenine; Serotonin; Age; Lipopolysaccharide

\section{INTRODUCTION}

Changes in tryptophan (TRP) metabolism play an important role in the brain-endocrine-immune system interaction that is hypothesized to be involved in the etiology and/or pathophysiology of major depression. Two main pathways metabolize TRP. One is the kynurenine (KYN) pathway, which is initiated by indoleamine 2, 3 -dioxygenase (IDO). The other is the serotonin (5-HT) pathway, which is initiated by the enzyme tryptophan hydroxylase (TPH).

An induction of IDO by inflammation may occur in depression [1]. Studies of depression induced by cytokine therapy have indicated that the severity of depressive symptoms is correlated with decreases in serum TRP and/or increases in KYN [2-4]. Immunological challenges such as exposure to proinflammatory cytokines (IL- 1, IFN- $\alpha$, IFN- $\gamma$, and TNF- $\alpha$ ) induce IDO activity [5-10], which metabolizes TRP to KYN, deprives TPH of its substrate, and may result in 5-HT depletion [11]. Because IDO can directly metabolize 5-HT [12], activated IDO may also decrease 5-HT levels [13]. Such 5-HT depletion is included in the monoamine hypothesis, a major etiological hypothesis for depression that proposes the existence of a relationship between decreases in monoamines in the brain and the onset and symptoms of depression [14-15].

According to the monocyte-T-lymphocyte hypothesis [16-17], IL-1 released from activated macrophages directly stimulates corticotropin-releasing hormone (CRH) release from the paraventricular nucleus (PVN) in the hypothalamus. Thus, the HPA-axis hyperactivity hypothesis, which proposes a relationship between HPAaxis hyperactivity and depression [18], closely relates to changes elicited by macrophage hyperactivity.

As noted above, changes in immunological activity have been incorporated into two main hypotheses about the etiology and pathophysiology of major depression. A shift in the balance between the KYN and 5-HT pathways to the KYN pathway may occur in the brain of patients with major depression [19]. However, patients ordinarily 
become depressed in response to adverse life events and/or the loss of social support (i.e., psychological and/or environmental factors), especially when such events or losses are encountered at an advanced age [20-21]. In order to investigate the shift in the balance in the TRP metabolism to the KYN pathway, the influence of such factors on TRP metabolism was investigated [22]. Specifically, a previously designed animal model [23-28] was applied to assess the influence of the following three risk factors on TRP metabolism: age, social isolation, and activation of the inflammation response system (IRS). The results showed that older age and social isolation shifted the balance between the KYN and 5-HT pathways to the 5-HT pathway, whereas novelty stress shifted the balance to the KYN pathway [22]. Further study revealed that immunological challenges such as intraperitoneal (i.p.) injection of lipopolysaccharide (LPS) shifted the balance to the KYN pathway [29]. These studies may confirm that psychological stressors, as well as immunological challenges, can shift the balance between the KYN and 5-HT pathways toward the KYN pathway, as expected. Although interactions between social isolation and systemic LPS injection on TRP metabolism in the brain have been observed [29], the influence of age on changes elicited by systemic LPS injection remains to be examined.

The aim of the present study was to clarify the influence of age on a shift in the balance between the KYN and 5-HT pathways elicited by i.p. injection of LPS. For this series, four brain regions were selected, the first three of which possess 5-HT nerve terminals: the prefrontal cortex, because it relates to behavioral motivation; the amygdala, because it relates to emotion; the hippocampus, because it regulates the HPA axis, and hyperactivity of this axis is closely related to the etiology and pathophysiology of depression; and the dorsal raphe nuclei, because they contain the cell bodies of 5-HT neurons and are the center of brain 5-HT synthesis.

\section{METHODOLOGY}

\subsection{Animals}

A total of 32 male-specific, pathogen-free (SPF) ICR mice were used in the present experiments. At 21 postnatal days (PND), the mice were housed in groups of 4-5 per cage, and they were reared for 4 weeks (younger group) or until they became 1 year old (older group). On the final day (Day 28 for the younger group, and 1 year old for the older group), the animals were further separated into two groups and were then given a $0 \mathrm{mg} / \mathrm{kg}$ or $1 \mathrm{mg} / \mathrm{kg}$ intraperitoneal (i.p.) injection of LPS diluted with saline. Thus, the mice were divided into 4 groups as follows: younger, LPS $0 \mathrm{mg} / \mathrm{kg}(\mathrm{n}=8)$; younger, LPS 1 $\mathrm{mg} / \mathrm{kg}(\mathrm{n}=8)$; older, LPS $0 \mathrm{mg} / \mathrm{kg}(\mathrm{n}=8)$; older, LPS 1 $\mathrm{mg} / \mathrm{kg}(\mathrm{n}=8)$.
Mice in the older group with apparent wounds were excluded from the study, because any such wound would itself likely have influenced the immune response, and in turn TRP metabolism. Although this selection process excluded apparently subordinate mice from the study, it was more important to avoid the potential influence of the immune responses associated with wounds on TRP metabolism.

The cages used in this series measured $21 \times 31 \times 13$ $\mathrm{cm}$. Cage exchange was performed 2 times per week. Food and water were provided ad libitum. The animals were kept on a 12-h light/dark cycle (lights on at $09.00 \mathrm{~h}$, off at $21.00 \mathrm{~h}$ ), and room temperature was maintained at $21-23^{\circ} \mathrm{C}$. All efforts were made to minimize both the number of animals used and the degree of their suffering. All experiments were conducted in accordance with the European Communities Council Directive of November 24, $1986(86 / 609 / \mathrm{EEC})$. The study was approved by the ethical committee of the Nagoya University Graduate School of Medicine.

\subsection{LPS Injection}

For the LPS injection, LPS from E. coli (Sigma, St. Louis, MO) was diluted with saline $(2 \mathrm{mg} / 10 \mathrm{ml})$ and i.p.-injected $(0 \mathrm{mg} / \mathrm{kg}$ or $1 \mathrm{mg} / \mathrm{kg}$ ) into the mice (saline only in the $0 \mathrm{mg} / \mathrm{kg}$ group). The time of LPS injection was between $11.00 \mathrm{~h}$ and $14.00 \mathrm{~h}$. The injection volume was $5 \mathrm{ml} / \mathrm{kg}$. After having received the LPS injection, the mice were returned to their rearing cages until brain sample preparation.

\subsection{Sample Preparation}

Four brain regions (prefrontal cortex, hippocampus, amygdala, and dorsal raphe nuclei) were dissected from the whole brain.

The mice were sacrificed by decapitation $4 \mathrm{~h}$ after the i.p.-injection of LPS; mice were decapitated under brief ether anesthesia. The brains were removed, and four brain regions were dissected out as quickly as possible on glass plates over ice [22]. The samples were weighed and treated with $1000 \mu \mathrm{l}$ of an ice-cold $0.2 \mathrm{M}$ trichloroacetic acid (TCA) solution containing $0.2 \mathrm{mM}$ sodium pyrosulfite, $0.01 \%$ EDTA-2Na, and $0.5 \mu \mathrm{M}$ isoproterenol (ISO) and 3-nitro-L-tyrosine (3-NTYR) as an internal standard per $100 \mathrm{mg}$ of wet tissue. The solution was sonicated and then centrifuged at $10,000 \mathrm{~g}$ for 20 min at $4^{\circ} \mathrm{C}$. The supernatant was filtered through a Millipore HV filter $(0.45 \mu \mathrm{m}$ pore size) and then subjected to both high-performance liquid chromatography (HPLC) with electrochemical detection (ECD) of 5-HT, and HPLC with fluorimetric detection (FD) of TRP as well as ultraviolet (UV) detection of KYN.

The standard solution was prepared using the abovementioned ice-cold $0.2 \mathrm{M}$ TCA solution containing $0.5-\mu \mathrm{M}$ internal standards (ISO, 3-NTYR), and the solu- 
tion concentrations were adjusted to $0.5 \mu \mathrm{M}$ for 5 -HT and $\mathrm{KYN}$, and to $10 \mu \mathrm{M}$ for TRP.

\subsection{HPLC Determination of 5-HT Levels in the Brain}

The levels of 5-HT in the brain extracts were measured by HPLC with ECD. The HPLC-ECD system employed here consisted of a CMA/200 autosampler (CMA/Microdialysis AB, Stockholm, Sweden), a micro LC pump (BAS, West Lafayette, IN), an LC-4C ECD (BAS), a Bio-Phase ODS-4 51-6034 column (4.0x110 mm; BAS), a CR-6A recorder (Shimadzu, Kyoto, Japan), an LC-26A vacuum degasser (BAS), and a CTO-10A column heater set at $35^{\circ} \mathrm{C}$ (Shimadzu). The mobile-phase solution consisted of $0.1 \mathrm{M}$ tartaric acid-0.1 M sodium acetate buffer (pH 3.2), containing $0.5 \mathrm{mM}$ EDTA-2Na, $555 \mu \mathrm{M}$ sodium 1-octane sulfonate, and 5\% acetonitrile. The flow rate was $700 \mu \mathrm{l} / \mathrm{min}$. The concentration of each compound was calculated by comparison with both internal (ISO) and external standards. The sensitivity of the 5-HT measurements was $150 \mathrm{f} \mathrm{mol}$.

\subsection{HPLC Determination of Brain Levels of TRP and KYN}

The levels of TRP and KYN were measured according to the methods of Widner and colleagues [30-31]. The HPLC pump was an LC-10AD (Shimadzu). For separation, reversed-phase column LiChroCART 55-4 cartridges filled with Purospher STAR Rp-18e $(55-\mathrm{mm}$ length, $3-\mu \mathrm{m}$ grain size) together with a reverse-phase LiChroCART 4-4 precolumn filled with Purospher STAR RP-18e (5- $\mu \mathrm{m}$ grain size; Merck, Rahway, NJ) were used. In this series, TRP was detected by RF-535 FD (Shimadzu) at an excitation wavelength of $285 \mathrm{~nm}$ and an emission wavelength of $365 \mathrm{~nm}$. KYN and 3-NTYR were detected by a SPD-10A UV-detector (Shimadzu) at a wavelength of $360 \mathrm{~nm}$. Both detectors were connected in a series to enable simultaneous measurement. The mobile-phase solution consisted of a 15-mM L-acetic acid-sodium acetate buffer, $\mathrm{pH}$ 4.0, containing $2.7 \%$ acetonitrile. The flow rate was 900 $\mu \mathrm{L} / \mathrm{min}$ at room temperature. The sensitivities of the TRP and KYN measurements were, respectively, $50 \mathrm{f}$ $\mathrm{mol}$ and $100 \mathrm{fmol}$.

\subsection{Statistical Analyses}

To examine differences in the levels of TRP, 5-HT, and $\mathrm{KYN}$, and in the ratios of KYN/TRP, 5-HT/TRP, and KYN/5-HT, two-way MANOVA (Wilks's lambda) for age and LPS was conducted on dependent measures in each brain region, followed by the Tukey-Kramer test. Data are presented as the means \pm S.E.M. All P values of less than 0.05 were accepted as significant.

\section{RESULTS}

\subsection{Prefrontal Cortex}

The results of the two-way MANOVA for age and LPS were as follows: age $(\mathrm{F}(6,23)=6.917 ; \mathrm{P}=0.0003)$ and LPS $(\mathrm{F}(6,23)=11.020 ; \mathrm{P}<0.0001)$ significantly altered the dependent measures. The interaction between age and $\operatorname{LPS}(\mathrm{F}(6,23)=3.810 ; \mathrm{P}=0.0088)$ was significant. The results of the post-hoc test are shown in Figure 1(a) and Table 1(a). Age significantly decreased the KYN/5-HT ratio ( $p<0.01$, Table 1(a)), whereas it did not alter that of KYN/TRP (Table 1(a)). Thus, age shifted the balance between KYN and 5-HT pathways toward the 5-HT pathway. Whereas LPS significantly increased the KYN/5-HT ratio ( $p<0.01$, Table1(a)), it did not alter the KYN/TRP ratio (Table 1(a)). Thus, LPS shifted the balance between the KYN and 5-HT pathways toward the KYN pathway.

\subsection{Hippocampus}

The results of the two-way MANOVA for age and LPS were as follows: age $(\mathrm{F}(6,23)=10.114 ; \mathrm{P}<0.0001)$ and LPS $(F(6,23)=10.393 ; P<0.0001)$ significantly altered the dependent measures. The interaction between age and LPS $(F(6,23)=3.676 ; \mathrm{P}=0.0105)$ was significant. The results of the post-hoc test are shown in Figure 1(b) and Table 1(b). Age significantly decreased the ratios of KYN/TRP $(\mathrm{p}<0.01$, Table $1(\mathbf{b}))$ and KYN $/ 5-H T(p<0.01$, Table 1(b). Thus, age shifted the balance between the KYN and 5-HT pathways to the 5-HT pathway. Although LPS significantly decreased the KYN/TRP ratio $(p<0.05$, Table 1(b)), it increased the KYN/5-HT ratio $(\mathrm{p}<0.01$, Table $1(\mathbf{b}))$. Thus, LPS shifted the balance between KYN and 5-HT pathways toward the KYN pathway.

\subsection{Amygdala}

The results of the two-way MANOVA for age and LPS were as follows: age $(\mathrm{F}(6,23)=13.776 ; \mathrm{P}<0.0001)$ and LPS $(F(6,23)=14.523 ; P<0.0001)$ significantly altered the dependent measures. The interaction between age and LPS $(F(6,23)=5.904 ; \mathrm{P}=0.0008)$ was significant. The results of the post-hoc test are shown in the Figure 1(c) and Table 1(c). Age significantly decreased the KYN/5-HT ratio $(\mathrm{p}<0.01$, Table $1(\mathrm{c}))$, whereas it did not alter the KYN/TRP ratio (Table 1(c)). Thus, age shifted the balance between the KYN and 5-HT pathways to the 5-HT pathway. Whereas LPS significantly increased the KYN/5-HT ratio $(\mathrm{p}<0.01$, Table $1(\mathbf{c}))$, it did not alter the KYN/TRP ratio (Table 1(c)). Thus, LPS shifted the balance between the KYN and 5-HT pathways toward the KYN pathway. 


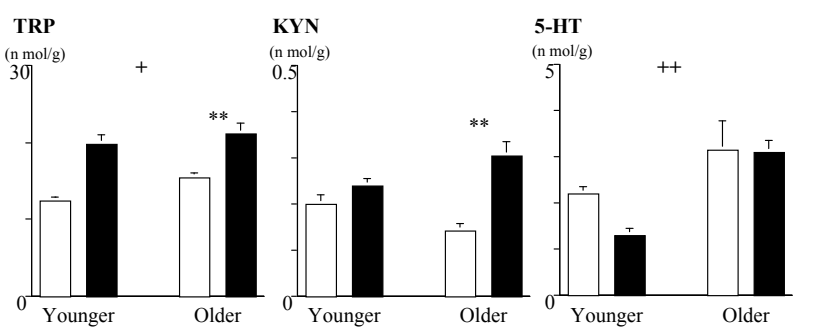

(a)

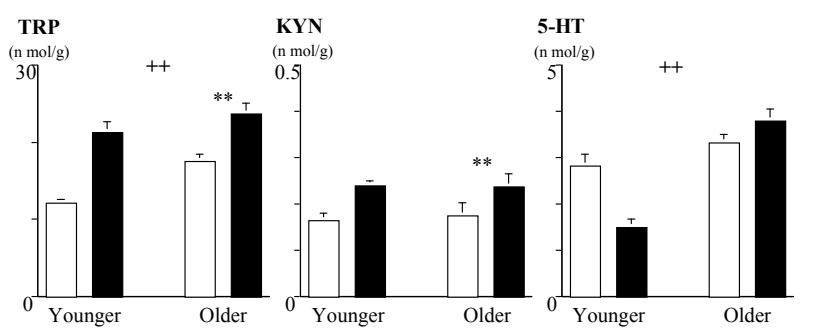

(c)

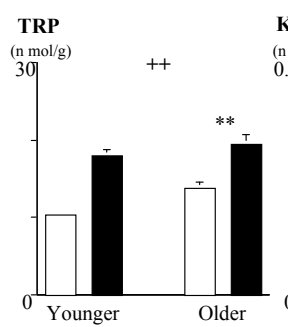

Older

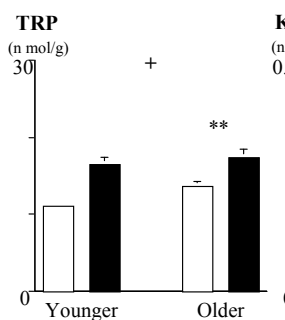

Older

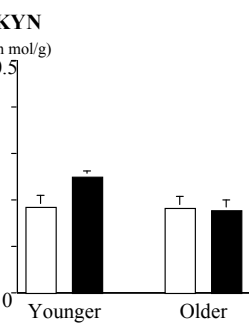

(b)

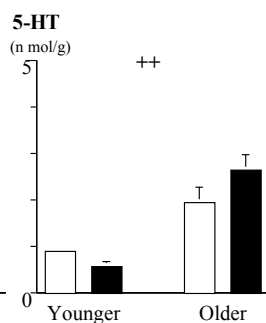

Figure 1. Changes in tryptophan (TRP), kynurenine (KYN), and serotonin (5-HT) levels elicited by age and LPS. Each bar indicates a group defined by age and LPS (n=8). Younger, younger group; Older, older group. White bar, LPS $0 \mathrm{mg} / \mathrm{kg}$; black bar, LPS $1 \mathrm{mg} / \mathrm{kg}$. Values are shown as means \pm S.E.M. The results of the Tukey-Kramer test for age and LPS are shown. Effects of age are shown:,$+ p$ $<0.05 ;++, \mathrm{p}<0.01$. Effects of LPS are shown: *, p < 0.05; **, p < 0.01. (a) Prefrontal cortex; (b) hippocampus; (c) amygdala; (d) dorsal raphe nuclei.

Table 1. Changes in the KYN/TRP, 5-HT/TRP, and KYN/5-HT ratios elicited by age and LPS. Eight animals were used in each group (as defined by age and LPS). Values are shown as means \pm S.E.M. The results of the Tukey-Kramer test for age and LPS are shown. Effects of age are shown:,$+ \mathrm{p}<0.05 ;++, \mathrm{p}<0.01$. Effects of LPS are shown: ${ }^{*}, \mathrm{p}<0.05 ; * *, \mathrm{p}<0.01$. (a) prefrontal cortex; (b) hippocampus; (c) amygdala; (d) dorsal raphe nuclei.

\begin{tabular}{|c|c|c|c|c|}
\hline \multicolumn{5}{|c|}{ (a) Prefrontal cortex } \\
\hline Age & LPS & KYN/TRP & 5-HT/TRP & KYN/5-HT \\
\hline Younger & $0 \mathrm{mg} / \mathrm{kg}$ & $0.016 \pm 0.002$ & $0.180 \pm 0.014$ & $0.093 \pm 0.011$ \\
\hline Younger & $1 \mathrm{mg} / \mathrm{kg}$ & $0.012 \pm 0.001$ & $0.068 \pm 0.010$ & $0.204 \pm 0.028$ \\
\hline Older & $0 \mathrm{mg} / \mathrm{kg}$ & $0.009 \pm 0.001$ & $0.211 \pm 0.046$ & $0.054 \pm 0.009$ \\
\hline Older & $1 \mathrm{mg} / \mathrm{kg}$ & $0.015 \pm 0.002$ & $0.150 \pm 0.015+, * *$ & $0.106 \pm 0.016++, * *$ \\
\hline \multicolumn{5}{|c|}{ (b) Hippocampus } \\
\hline Age & LPS & KYN/TRP & 5-HT/TRP & KYN/5-HT \\
\hline Younger & $0 \mathrm{mg} / \mathrm{kg}$ & $0.018 \pm 0.002$ & $0.089 \pm 0.008$ & $0.213 \pm 0.035$ \\
\hline Younger & $1 \mathrm{mg} / \mathrm{kg}$ & $0.014 \pm 0.001$ & $0.033 \pm 0.006$ & $0.500 \pm 0.079$ \\
\hline Older & $0 \mathrm{mg} / \mathrm{kg}$ & $0.013 \pm 0.002$ & $0.144 \pm 0.023$ & $0.107 \pm 0.018$ \\
\hline Older & $1 \mathrm{mg} / \mathrm{kg}$ & $0.009 \pm 0.001++, *$ & $0.142 \pm 0.025++$ & $0.079 \pm 0.019++, * *$ \\
\hline \multicolumn{5}{|c|}{ (c) Amygdala } \\
\hline Age & LPS & KYN/TRP & 5-HT/TRP & KYN/5-HT \\
\hline Younger & $0 \mathrm{mg} / \mathrm{kg}$ & $0.013 \pm 0.001$ & $0.235 \pm 0.024$ & $0.060 \pm 0.006$ \\
\hline Younger & $1 \mathrm{mg} / \mathrm{kg}$ & $0.011 \pm 0.001$ & $0.070 \pm 0.007$ & $0.175 \pm 0.022$ \\
\hline Older & $0 \mathrm{mg} / \mathrm{kg}$ & $0.010 \pm 0.002$ & $0.195 \pm 0.017$ & $0.056 \pm 0.012$ \\
\hline Older & $1 \mathrm{mg} / \mathrm{kg}$ & $0.010 \pm 0.001$ & $0.163 \pm 0.011 * *$ & $0.062 \pm 0.005++, * *$ \\
\hline \multicolumn{5}{|c|}{ (d) Dorsal raphe nuclei } \\
\hline Age & LPS & KYN/TRP & 5-HT/TRP & KYN/5-HT \\
\hline Younger & $0 \mathrm{mg} / \mathrm{kg}$ & $0.021 \pm 0.004$ & $0.119 \pm 0.023$ & $0.193 \pm 0.029$ \\
\hline Younger & $1 \mathrm{mg} / \mathrm{kg}$ & $0.016 \pm 0.002$ & $0.073 \pm 0.007$ & $0.223 \pm 0.028$ \\
\hline Older & $0 \mathrm{mg} / \mathrm{kg}$ & $0.013 \pm 0.002$ & $0.152 \pm 0.012$ & $0.133 \pm 0.052$ \\
\hline Older & $1 \mathrm{mg} / \mathrm{kg}$ & $0.009 \pm 0.001++$ & $0.148 \pm 0.021++$ & $0.071 \pm 0.017++$ \\
\hline
\end{tabular}




\subsection{Dorsal Raphe Nuclei}

The results of the two-way MANOVA for age and LPS were as follows: age $(\mathrm{F}(6,23)=10.978 ; \mathrm{P}<0.0001)$ and LPS $(\mathrm{F}(6,23)=5.751 ; \mathrm{P}=0.0009)$ significantly altered the dependent measures. The interaction between age and LPS $(F(6,23)=1.757 ; P=0.1529)$ was not significant. The results of the post-hoc test are shown in Figure 1(d) and Table 1(d). Age significantly decreased the KYN/ TRP $(p<0.01$, Table 1(d)) and the KYN/5-HT $(p<0.01$, Table 1(d)) ratios. Thus, age shifted the balance between the KYN and 5-HT pathways to the 5-HT pathway. Moreover, LPS did not alter the KYN/TRP, 5-HT/TRP, or KYN/5-HT ratios (Table 1(d)). Thus, LPS did not shift the balance between the KYN and 5-HT pathways.

\section{DISCUSSION}

In the present study, the influences of age on the balance between the KYN and 5-HT pathways of brain TRP metabolism were investigated. This balance has been known to shift in favor of the KYN pathway in response to a systemic injection of LPS.

\subsection{Effects of Age on Brain TRP Metabolism}

Age shifted the balance between the KYN and 5-HT pathways to the 5-HT pathway, as was expected based on the results of our previous study [22]. Animal experiments have yielded controversial findings on the effects of age on KYN pathway activity. One previous study suggested that age activates the KYN pathway, especially as regards the synthesis of kynurenic acid (KYNA), a neuroprotective product of the KYN pathway [32]. However, another study suggested that age reduces IDO activity in the liver, kidneys, and small intestines of rats [33]. In a human study, Alzheimer's disease patients and age-matched controls exhibited a decrease in plasma TRP levels and an increase in the KYN/TRP ratio, as compared to those of a younger control group [34]. Furthermore, the serum KYN/TRP ratio in healthy individuals was found to increase with older age [35], and the serum KYN/TRP ratio in nonagenarians was significantly higher than that of controls [36]. As the serum KYN/TRP ratio has been found to consistently rise with increasing age, it serves as a marker of IDO activity in humans. In the present study, no evidence of activation of the KYN pathway elicited by age was found. Instead, the KYN/TRP ratio decreased in the hippocampus and dorsal raphe nuclei, as age was associated with increased TRP levels, while KYN levels remained unaltered or to some extent decreased. Apparently, increases in age are associated with increases in brain 5-HT levels. The present findings regarding the abovementioned shifts are based primarily on an increase in 5-HT levels. However, previous studies have reported increases [37-38], no change [39-40], or even decreases [41-42] in 5-HT levels elicited by age. Thus, the influence of age on brain 5-HT levels remains controversial. A previous rat-model study revealed a decrease in the 5 -HT turnover ratio elicited by age [24], and therefore decreased 5-HT turnover is suspected to have been the cause of observed increases in 5-HT levels. Although the effects of age on TRP metabolism were discussed in a previous study, the age of the mice used (6 months) in that study was not sufficient to investigate the effects of age [22]. In the present study, older (1-year-old) mice were used in order to clarify this point. The results of our previous study regarding the effects of age on TRP metabolism were confirmed here. Although the age of the mice in the present study may still have been insufficient for studying the effects of age, the older (1-year-old) mice did exhibit more of a shift in the balance between the KYN and 5-HT pathways to the 5-HT pathway, as well as more of a decrease in the KYN/TRP ratio in the hippocampus and dorsal raphe nuclei, and more of an increase in the 5-HT/TRP ratio in all regions (except for the amygdala), than the shift observed in young adult (6-month-old) mice (i.e., increased 5 -HT/TRP ratio in the prefrontal cortex and hippocampus) [22].

\subsection{Effects of Systemic LPS Administration on Brain TRP Metabolism}

A shift in the balance between the KYN and 5-HT pathways to the KYN pathway after LPS exposure was observed, as LPS increased brain TRP and KYN levels. These results confirmed previously reported data regarding the influence of LPS on TRP metabolism [29]. The putative mechanisms of these changes elicited by LPS are as follows.

The elevation in TRP is associated with the following mechanisms. One mechanism regulating brain TRP levels is the free fatty acid (FFA) levels induced by the sympathetic nerve system. The activated sympathetic nerve increases plasma FFA, which binds to albumin. The albumin bound to FFA reduces its affinity for TRP [43], resulting in an increase in free plasma TRP levels [44-45]. The other mechanism regulating brain TRP levels is the competition between TRP and the so-called "large neutral" amino acids (LNAA), which include aromatic amino acids and branched-chain amino acids (BCAA), at uptake sites at the blood-brain barrier (BBB). Elevation of the plasma TRP/LNAA ratio regulates TRP uptake activity at the BBB [45-47]. Thus, LPS may increase plasma FFA, which would in turn bind to albumin and reduce its affinity for TRP, resulting in an increase in free plasma TRP. Due to the increase in the TRP/LNAA ratio at $\mathrm{BBB}$ amino acid-uptake sites, LPS may lead to increase brain TRP levels [19].

In the present study, systemic LPS administration ac- 
tually increased brain KYN levels. Systemic LPS administration is known to increase both brain [48] and peripheral [49] IDO activity. Because KYN is thought to enter the brain by the same system that transports TRP and other large amino acids [50], blood KYN may enter the brain. In fact, $60 \%$ of the total KYN pool in the normal brain is derived from blood [51]. In gerbils, $78 \%$ of the brain KYN is derived from blood, whereas brain KYN is derived exclusively from the blood after systemic LPS injection [52]. Thus, most of the increase in brain KYN elicited by systemic LPS injection in these studies may have originated from outside of the brain. However, in the present study, immune-activated brain microglia and/or astrocytes may have synthesized KYN, thus accounting for a small portion of the increase in brain KYN. Here, the systemic administration of LPS did not directly increase the brain KYN/TRP ratio. It is likely that the increase in free TRP in the plasma preceded the newly synthesized KYN by peripherally activated IDO. A recent study has indicated that the effects of i.p. injection of LPS on the mRNA expression of IDO in the hippocampus and hypothalamus reached a peak at $6 \mathrm{~h}$ after injection [53]. Another study has suggested that i.p.-LPS injection actually increased the KYN/TRP ratio in the whole brain at $28 \mathrm{~h}$ following injection [54]. Thus, the brain KYN/TRP ratio at $4 \mathrm{~h}$ after LPS injection may not have reflected the maximum elevation of IDO activity elicited by LPS.

In the present study, LPS did not significantly reduce the level of 5-HT, although a previous study revealed a reduction in 5-HT levels with the administration of LPS [29]. Because LPS clearly decreased 5-HT levels in all brain regions except the dorsal raphe nuclei of the younger group (i.e., the Tukey-Kramer test showed significant decreases, data not shown), the unchanged 5-HT levels may be primarily attributable to a lack of LPSalteration of 5-HT levels in the older group of mice. The mechanisms by which different 5-HT levels are produced at different ages post-LPS administration remain to be elucidated in the future studies.

Because novelty stress was also found to shift the balance to the KYN pathway [22], these results suggest an overlap between the neurochemical changes elicited by stressor and immune challenges, as has been frequently noted elsewhere [55-59]. The activation of the IRS by either direct immunological activation or psychological stress activates IDO and shifts the balance to the KYN pathway [19].

\subsection{Influence of Age on Changes in Brain TRP Metabolism Elicited by Systemic LPS Administration}

Although there were clear effects of age on TRP metabolism that led to a shifting of the balance between KYN and 5-HT pathways to the 5-HT pathway, the precise underlying mechanisms of these effects remain unknown. However, age was found to shift the balance of TRP metabolism in a direction opposed to that elicited by LPS. In other words, age suppressed the changes in TRP metabolism elicited by LPS (i.e., inhibited a shift in the balance between the 5-HT and KYN pathways to the KYN pathway). Suppression of this shift is potentially important in terms of the etiology of psychiatric disorders such as delirium elicited by physical illness, as well as depression in the elderly [60-61], because elderly people are known to be more vulnerable than younger people to delirium elicited by systemic inflammation [62]. It is likely that the combined inhibition of stress and immunological responses is closely related to such vulnerability, because sufficient-but not prolonged-responses to stress and immunological challenge are needed to quickly recover from these stimuli. Further evidence will still be needed to clarify this point.

\subsection{Limitations of the Study Design}

An important limitation of the present study was that the immune system activation elicited by a single systemic injection of LPS was acute, not chronic. Because the type of immunological activation in cases of depression is thought to be chronic rather than acute, the behavioral changes elicited by acute immune system activation has been referred to as "sickness behavior" and is not directly comparable to depression. This limitation was present in the protocol design of the present study. However, a recent study using mice demonstrated that the peripheral administration of LPS activated IDO, and culminated in a distinct depressive-like behavioral syndrome, as measured by increased duration of immobility in both a forced-swim test at $24 \mathrm{~h}$ and a tail-suspension test at $28 \mathrm{~h}$ following the administration of LPS [54]. Because the present study was designed independently of this new study, only the early-phase changes in TRP metabolism (i.e., $4 \mathrm{~h}$ after LPS injection) were examined. Thus, the time course of changes in TRP metabolism until the appearance of a depressive-like behavioral syndrome was not investigated. Although acute systemic LPS injection increased brain TRP levels, it is believed that depression is accompanied by lowered plasma and brain TRP levels [63-64], and depletion of TRP by the administration of high doses of BCAAs may induce depression in some vulnerable subjects and depressed subjects who are in remission [65]. The discrepancy between the TRP increase elicited by a single systemic administration of LPS and TRP reduction seen in cases of depression suggest that the present results do not directly correspond with the pathophysiology of depression.

\section{CONCLUSIONS}

The influences of age and the acute systemic administra- 
tion of LPS on the balance between the KYN and 5-HT pathways of brain TRP metabolism were investigated. Whereas age shifted the balance of TRP metabolism to the 5-HT pathway, the acute systemic administration of LPS shifted the balance of these pathways in favor of the KYN pathway. Age countered the effects of LPS. Thus, age attenuated the normal, sufficient immunological brain TRP-metabolism response, which is thought to be an essential part of the normal stress response. These effects will be important to consider as they might be related to the etiology of psychiatric disorders in elderly people.

\section{ACKNOWLDGEMENTS}

We would like to thank Mr. Ogiso, a technician in the Division of Experimental Animals, Nagoya University, who kindly advised us regarding the protocol for the animal experiments. This research was supported by a grant from the Japanese Ministry of Health, Labor, and Welfare for Comprehensive Research on Aging and Health.

\section{REFERENCES}

[1] Maes, M., Scharpe, S., Meltzer, H.Y., Okayli, G., Bosmans, E., D'Hondt, P., Vanden Bossche, B.V. and Cosyns, P. (1994) Increased neopterin and interferon-gamma secretion and lower availability of L-tryptophan in major depression: further evidence for an immune response. Psychiatry Research, 54, 143-160.

[2] Bonaccorso, S., Marino, V., Puzella, A., Pasquini, M., Biondi, M., Artini, M., Almerighi, C., Verkerk, R., Meltzer, H. and Maes, M. (2002) Increased depressive ratings in patients with hepatitis $\mathrm{C}$ receiving interferon-alpha- based immunotherapy are related to interferon-alpha- induced changes in the serotonergic system. Journal of Clinical Psychopharmacology, 22, 86-90.

[3] Capuron, L., Ravaud, A., Neveu, P.J., Miller, A.H., Maes, M. and Dantzer, R. (2002) Association between decreased serum tryptophan concentrations and depressive symptoms in cancer patients undergoing cytokine therapy. Molecular Psychiatry, 7, 468-473.

[4] Capuron, L., Neurauter, G., Musselman, D.L., Lawson, D.H., Nemeroff, C.B., Fuchs, D., Miller and A.H. (2003) Interferon-alpha-induced changes in tryptophan metabolism. relationship to depression and paroxetine treatment. Biological Psychiatry, 54, 906-914.

[5] Stone, T.W. and Darlington, L.G. (2002) Endogenous kynurenines as targets for drug discovery and development. Nature Reviews Drug Discovery, 1, 609-620.

[6] Konsman, J.P., Parnet, P. and Dantzer, R. (2002) Cytokine-induced sickness behaviour: mechanisms and implications. Trends in Neurosciences, 25, 154-159.

[7] Widner, B., Laich, A., Sperner-Unterweger, B., Ledochowski, M. and Fuchs, D. (2002) Neopterin production, tryptophan degradation, and mental depression-what is the link? Brain, Behavior, and Immunity, 16, 590-595.

[8] Moffett, J.R. and Namboodiri, M.A. (2003) Tryptophan and the immune response. Immunology and Cell Biology, 81, 247- 265 .
[9] Myint, A.M. and Kim, Y.K. (2003) Cytokine-serotonin interaction through IDO: A neurodegeneration hypothesis of depression. Medical Hypotheses, 61, 519-525.

[10] Wichers, M.C. and Maes, M. (2004) The role of indoleamine 2,3-dioxygenase (IDO) in the pathophysiology of interferon-alpha-induced depression. Journal of Psychiatry and Neuroscience, 29, 11-17.

[11] Dantzer, R., Wollman, E., Vitkovic, L. and Yirmiya, R. (1999) Cytokines and depression: Fortuitous or causative association? Molecular Psychiatry, 4, 328-332.

[12] Shimizu, T., Nomiyama, S., Hirata, F. and Hayaishi, O. (1978) Indoleamine 2, 3-dioxygenase. Purification and some properties. Journal of Biological Chemistry, 253, $4700-4706$.

[13] Muller, N. and Schwarz, M.J. (2007) The immune- mediated alteration of serotonin and glutamate: Towards an integrated view of depression. Molecular Psychiatry, 12, 988-1000.

[14] Delgado, P.L. (2004) How antidepressants help depression: Mechanisms of action and clinical response. Journal of Clinical Psychopharmacology, 65(4-Supplemnt), 25-30.

[15] Owens, M.J. (2004) Selectivity of antidepressants: From the monoamine hypothesis of depression to the SSRI revolution and beyond. Journal of Clinical Psychopharmacology 65 (4-Supplement), 5-10.

[16] Smith, R.S. (1991) The macrophage theory of depression. Medical Hypotheses, 35, 298-306.

[17] Maes, M., Smith, R. and Scharpe, S. (1995) The monocyte-T-lymphocyte hypothesis of major depression. Psychoneuroendocrinology, 20, 111-116.

[18] Schule, C. (2007) Neuroendocrinological mechanisms of actions of antidepressant drugs. Journal of Neuroendocrinology, 19, 213-226.

[19] Miura, H., Ozaki, N., Sawada, M., Isobe, K., Ohta, T. and Nagatsu, T. (2008b) A link between stress and depression: Shifts in the balance between the kynurenine and serotonin pathways of tryptophan metabolism and the etiology and pathophysiology of depression. Stress, 11, 198-209.

[20] Kendler, K.S., Kessler, R.C., Neale, M.C., Heath, A.C. and Eaves, L.J. (1993) The prediction of major depression in women: toward an integrated etiologic model. American Journal of Psychiatry, 150, 1139-1148.

[21] Paykel, E.S. (1994) Life events, social support and depression. Acta Psychiatrica Scandinavica, (377 Supplement), 50-58.

[22] Miura, H., Ozaki, N., Shirokawa, T. and Isobe, K. (2008a) Changes in brain tryptophan metabolism elicited by ageing, social environment, and psychological stress in mice. Stress, 11, 160-169.

[23] Miura, H., Qiao, H. and Ohta, T. (2002a) Influence of aging and social isolation on changes in brain monoamine turnover and biosynthesis of rats elicited by novelty stress. Synapse, 46, 116-124.

[24] Miura, H., Qiao, H. and Ohta, T. (2002b) Attenuating effects of the isolated rearing condition on increased brain serotonin and dopamine turnover elicited by novelty stress. Brain Research, 926, 10-17.

[25] Miura, H., Qiao, H., Kitagami, T. and Ohta, T. (2004) Fluvoxamine, a selective serotonin reuptake inhibitor, suppresses tetrahydrobiopterin in the mouse hippocampus. Neuropharmacology, 46, 340-348.

[26] Miura, H., Qiao, H., Kitagami, T., Ohta, T. and Ozaki, N. 
(2005a) Effects of fluvoxamine on levels of dopamine, serotonin, and their metabolites in the hippocampus elicited by isolation housing and novelty stress in adult rats. International Journal of Neuroscience, 115, 367-378.

[27] Miura, H., Qiao, H., Kitagami, T., Ohta, T. and Ozaki, N. (2005b) Fluvoxamine, a selective serotonin reuptake inhibitor, suppresses tetrahydrobiopterin levels and dopamine as well as serotonin turnover in the mesoprefrontal system of mice. Psychopharmacology (Berl) 177, 307314.

[28] Miura, H., Kitagami, T. and Ozaki, N. (2007) Suppressive effect of paroxetine, a selective serotonin uptake inhibitor, on tetrahydrobiopterin levels and dopamine as well as serotonin turnover in the mesoprefrontal system of mice. Synapse, 61, 698-706.

[29] Miura. H., Shirokawa, T., Isobe, K. and Ozaki, N. (2009) Shifting the balance of brain tryptophan metabolism elicited by isolation housing and systemic administration of lipopolysaccharide in mice. Stress, 12, 206-214.

[30] Widner, B., Werner, E.R., Schennach, H., Wachter, H. and Fuchs, D. (1997) Simultaneous measurement of serum tryptophan and kynurenine by HPLC. Clinical Chemistry, 43, 2424-2426.

[31] Laich, A., Neurauter, G., Widner, B. and Fuchs, D. (2002) More rapid method for simultaneous measurement of tryptophan and kynurenine by HPLC. Clinical Chemistry, 48, 579- 581.

[32] Gramsbergen, J.B., Schmidt, W., Turski, W.A. and Schwarcz, R. (1992) Age-related changes in kynurenic acid production in rat brain. Brain Research, 588, 1-5.

[33] Comai, S., Costa, C.V.L., Ragazzi, E., Bertazzo, A. and Allegri, G. (2005) The effect of age on the enzyme activities of tryptophan metabolism along the kynurenine pathway in rats. Clinica Chimica Acta, 360, 67-80.

[34] Widner, B., Leblhuber, F., Walli, J., Tilz, G.P., Demel, U. and Fuchs, D. (2000) Tryptophan degradation and immune activation in Alzheimer's disease. Journal of Neural Transmission, 107, 343-353.

[35] Frick, B., Schroecksnadel, K., Neurauter, G., Leblhuber, F. and Fuchs, D. (2004) Increasing production of homocysteine and neopterin and degradation of tryptophan with older age. Clinical Biochemistry, 37, 684-687.

[36] Pertovaara, M., Rhaitala, A., Lehtimaki, T., Karhunen, P.J., Oja, S.S., Jylha, M., Hervonen, A. and Hurme, M. (2006) Indoleamine 2, 3-dioxygenase activity in nonagenarians is markedly increased and predicts mortality. Mechanisms of Ageing and Development, 127, 497-499.

[37] Santiago, M., Machado, A., Reinoso-Suarez, F. and Cano, J. (1988) Changes in biogenic amines in rat hippocampus during development and aging. Life Sciences, 42, 2503-2508.

[38] Delion, S., Chalon, S., Guilloteau, D., Lejeune, B., Besnard, J.C. and Durand, G. (1997) Age-related changes in phospholipid fatty acid composition and monoaminergic neurotransmission in the hippocampus of rats fed a balanced or an n-3 polyunsaturated fatty acid-deficient diet. Journal of Lipid Research, 38, 680-689.

[39] Ponzio, F., Calderini, G., Lomuscio, G., Vantini, G., Toffano, G. and Algeri, S. (1982) Changes in monoamines and their metabolite levels in some brain regions of aged rats. Neurobiology of Aging, 3, 23-29.

[40] Lee, J.M., Ross, E.R., Gower, A., Paris, J.M., Martensson,
R. and Lorens, S.A. (1994) Spatial learning deficits in the aged rat: neuroanatomical and neurochemical correlates. Brain Research Bulletin, 33, 489-500.

[41] Gozlan, H., Daval, G., Verge, D., Spampinato, U., Fattaccini, C.M., Gallissot, M.C., el Mestikawy, S. and Hamon, M. (1990) Aging associated changes in serotoninergic and dopaminergic pre- and postsynaptic neurochemical markers in the rat brain. Neurobiology of Aging, 11, 437-449.

[42] Luine, V., Bowling, D. and Hearns, M. (1990) Spatial memory deficits in aged rats: contributions of monoaminergic systems. Brain Research, 537, 271-278.

[43] McMenamy, R.H. (1965) Binding of indole analogues to human serum albumin. Effects of fatty acids. Journal of Biological Chemistry, 240, 4235-4243.

[44] Curzon, G., Friedel, J. and Knott, P.J. (1973) The effect of fatty acids on the binding of tryptophan to plasma protein. Nature, 242, 198-200.

[45] Struder, H.K., Hollmann, W., Platen, P., Wostmann, R., Weicker, H. and Molderings, G.J. (1999) Effect of acute and chronic exercise on plasma amino acids and prolactin concentrations and on $[3 \mathrm{H}]$ ketanserin binding to serotonin2A receptors on human platelets. European journal of applied physiology and occupational physiology, 79, 318-324.

[46] Fernstrom, J.D. and Wurtman, R.J. (1972) Brain serotonin content: physiological regulation by plasma neutral amino acids. Science, 178, 414-416.

[47] Chaouloff, F. (1997) Effects of acute physical exercise on central serotonergic systems. Medicine \& Science in Sports \& Exercise, 29, 58-62.

[48] Saito, K., Markey, S.P. and Heyes, M.P. (1992) Effects of immune activation on quinolinic acid and neuroactive kynurenines in the mouse. Neuroscience, 51, 25-39.

[49] Heyes, M.P., Saito, K., Chen, C.Y., Proescholdt, M.G., Nowak, T.S. Jr., Li, J., Beagles, K.E., Proescholdt, M.A., Zito, M.A., Kawai, K. and Markey, S.P. (1997) Species heterogeneity between gerbils and rats: quinolinate production by microglia and astrocytes and accumulations in response to ischemic brain injury and systemic immune activation. Journal of Neurochemistry, 69, 1519-1529.

[50] Fukui, S., Schwarcz, R., Rapoport, S.I., Takada, Y. and Smith, Q.R. (1991) Blood-brain barrier transport of kynurenines: implications for brain synthesis and metabolism. Journal of Neurochemistry, 56, 2007-2017.

[51] Gal, E.M. and Sherman, A.D. (1980) L-kynurenine: its synthesis and possible regulatory function in brain. Petrochemical Research, 5, 223-239.

[52] Kita, T., Morrison, P.F., Heyes, M.P. and Markey, S.P. (2002) Effects of systemic and central nervous system localized inflammation on the contributions of metabolic precursors to the L-kynurenine and quinolinic acid pools in brain. Journal of Neurochemistry, 82, 258-268.

[53] Andre, C., O’Connor, J.C., Kelly, K.W., Lestage, J., Danzer, R. and Castanon, N. (2008) Spatio-temporal differences in the profile of murine brain expression expression of proinflammatory cytokines and indoleamine 2, 3-dioxygenase in response to peripheral lipopolysaccharide administration. Journal of Neuroimmunology, 200, 90-99.

[54] O'Connor, J.C., Lawson, M.A., Andre, C., Moreau, M., Lestage, J., Castanon, N., Kelly, K.W. and Dantzer, R. (2009) Lipopolysaccharide-induced depressive-like be- 
havior is mediated by indoleamine 2,3-dioxygenase activation in mice. Molecular Psychiatry, 14, 511-522.

[55] Minami, M., Kuraishi, Y., Yamaguchi, T., Nakai, S., Hirai, Y. and Satoh, M. (1991) Immobilization stress induces interleukin-1 beta mRNA in the rat hypothalamus. $\mathrm{Neu}$ roscience Letters, 123, 254-256.

[56] Schulte, H.M., Bamberger, C.M., Elsen, H., Herrmann, G., Bamberger, A.M. and Barth, J. (1994) Systemic interleukin-1 alpha and interleukin-2 secretion in response to acute stress and to corticotropin-releasing hormone in humans. European Journal of Clinical Investigation, 24, 773-777.

[57] Shintani, F., Nakaki, T., Kanba, S., Sato, K., Yagi, G., Shiozawa, M., Aiso, S., Kato, R. and Asai, M. (1995) Involvement of interleukin-1 in immobilization stress-induced increase in plasma adrenocorticotropic hormone and in release of hypothalamic monoamines in the rat. Journal of Neuroscience, 15, 1961-1970.

[58] Maes, M., Song, C., Lin, A., De Jongh, R., Van Gastel, A., Kenis, G., Bosmans, E., De Meester, I., Benoy, I., Neels, H., Demedts, P., Janca, A., Scharpe, S. and Smith, R.S. (1998) The effects of psychological stress on humans: increased production of pro-inflammatory cytokines and a Th1-like response in stress-induced anxiety. Cytokine, 10, 313-318.

[59] Nguyen, K.T., Deak, T., Owens, S.M., Kohno, T., Fleshner,
M., Watkins, L.R. and Maier, S.F. (1998) Exposure to acute stress induces brain interleukin-1beta protein in the rat. Journal of Neuroscience, 18, 2239-2246.

[60] Burns, A., Gallagley, A. and Byrne, J. (2004) Delirium. Journal of Neurology Neurosurgery and Psychiatry, 75, 362-367.

[61] Inouye, S.K., Studenski, S., Tinetti, M.E. and Kuchel, G.A. (2007) Geriatric syndromes: clinical, research, and policy implications of a core geriatric concept. Journal of the American Geriatrics Society, 55, 780-791.

[62] Fong, T.G., Tulebaev, S. R. and Inoue, S.K. (2009) Delirium in elderly adults: diagnosis, prevention and treatment. Nature Reviews Neurology, 5, 210-220.

[63] Maes, M., Meltzer, H.Y., Scharpe, S., Bosmans, E., Suy, E., De Meester, I., Calabrese, J. and Cosyns, P. (1993) Relationships between lower plasma L-tryptophan levels and immune-inflammatory variables in depression. Psychiatry Research, 49, 151-165.

[64] Maes, M., Wauters, A., Verkerk, R., Demedts, P., Neels, H., Van Gastel, A., Cosyns, P., Scharpe, S. and Desnyder, R. (1996) Lower serum L-tryptophan availability in depression as a marker of a more generalized disorder in protein metabolism. Neuropsychopharmacology, 15, 243-251.

[65] Van der Does, A.J. (2001) The effects of tryptophan depletion on mood and psychiatric symptoms. Journal of Affective Disorders, 64, 107-119. 